\title{
Review
}

\section{Analgesic Action of Acupuncture and Moxibustion: A Review of Unique Approaches in Japan}

\section{Kaoru Okada and Kenji Kawakita}

Department of Physiology, Meiji University of Oriental Medicine, Hiyoshi-cho, Nantan-city, Kyoto 629-0392, Japan

\begin{abstract}
The mechanism of acupuncture analgesia (AA) is one of the most widely researched topics in complementary and alternative medicine (CAM) based on modern medical methodology. Endogenous opioid-mediated mechanisms of acupuncture have been well established since the 1970s. In this review, we have covered the progress of AA research by Japanese investigators. In particular, we have reviewed the physiological basis of analgesic effects induced by acupuncture and moxibustion, including the actions of endogenous opioid and diffuse noxious inhibitory controls (DNICs), and the afferent fibers participating in acupuncture and moxibustion stimuli are discussed.
\end{abstract}

Keywords: afferent fibers-diffuse noxious inhibitory control (DNIC)-endogenous opioids

\section{Introduction}

At early stages of acupuncture research in Japan, neural mechanisms were studied by many neurophysiologists. They demonstrated various neural connections in the central nervous system including ascending and descending spinal pathways and the involvement of endogenous opioids produced by low-frequency electro-acupuncture (EA) stimulation. On the other hand, recent reports indicate that in pathological conditions, the endogenous opioids act in peripheral tissues via the immune system. These opioid-mediated analgesic actions take time to develop and feature long-lasting effects. In contrast, DNIC is an analgesic phenomenon and is considered another pain inhibitory mechanism of acupuncture. Nociceptive discharges or reflexes are rapidly suppressed by acupuncture and moxibustion. Opioid and non-opioid mediated analgesic mechanisms may depend on the frequency of the afferent discharges activated by acupuncture and moxibustion stimulation.

For reprints and all correspondence: Kaoru Okada, Department of Physiology, Meiji University of Oriental Medicine, Hiyoshi-cho, Nantan-city, Kyoto 629-0392, Japan. Tel: +81-771-72-1181 (272); Fax: +81-771-72-0326; E-mail: k_okada@meiji-u.ac.jp

\section{Background on Scientific Research of Acupuncture in Japan}

Among the numerous complementary and alternative medicine (CAM) therapies, acupuncture and moxibustion are the most widely accepted as useful therapies for the treatment of acute and chronic pain. Analgesic mechanisms of acupuncture have been clarified by numerous studies that have demonstrated the roles of endogenous opioid peptides and various neurotransmitters in the central nervous system (1-5). Scientific studies of acupuncture in China using modern technology began in the late 1970s, and suggested that chemical mediators were involved in acupuncture analgesia (AA) $(6,7)$.

Numerous reports regarding the so-called miracle successes of various surgical operations under acupuncture needling in China surprised clinicians. At that time, the majority of Japanese medical doctors could not believe the successes and they assumed them to be a result of strong placebo effects as all subjects held copies of Mao Tse-Tung's Little Red Book in their photographs. It is unfortunate that acupuncture and moxibustion have been somewhat ignored in the modern medical system in Japan after the Meiji era (1868-1912, 44 years), that licensed acupuncture and moxibustion specialists have been educated outside of medical schools and that 
the majority of acupuncture treatments have been performed in private acupuncture clinics and not in hospitals. The basic mechanisms of AA were initially introduced by Aikawa (8). He reported the results of animal experiments conducted by Professor Chang, the world-famous neurophysiologist at the Shanghai Research Institute in China (9). The possible role of the centromedian (CM) nucleus of the thalamus in AA phenomena was clearly demonstrated in experimental animals, and this article promoted basic research of AA in Japan as it clearly demonstrated that AA is not a placebo phenomenon.

On the other hand, acupuncture and moxibustion therapies in Japan have their own long history and they have developed somewhat differently from Chinese procedures. One of the most important innovations was the development of a guide tube through which fine acupuncture needles by Waichi Sugiyama (1610-1694) could be inserted. In that era, acupuncturists frequently used fine needles made from gold and silver, which were very soft, and this made it quite difficult for them to penetrate the skin. The guide tube facilitated penetration of the skin without any painful sensation. The non-painful penetration and production of a gentle de-chi sensation are considered to be very important to the Japanese acupuncturist. Yano et al. (10) reported that electro-acupuncture (EA) at low frequency $(2 \mathrm{~Hz})$ accompanied with a comfortable $d e$-chi sensation increased the $\alpha$ - and $\theta$-waves in EEG activities and suggested the importance of the relaxation effect in clinical acupuncture treatments. In this brief review, we introduce the progress of AA research in Japan.

In particular, we have reviewed the physiological basis of analgesic effects induced by acupuncture and moxibustion including: (i) the actions of endogenous pain inhibitory mechanisms including the endogenous opioids, (ii) diffuse noxious inhibitory controls (DNICs), (iii) receptors and afferent pathways for acupuncture and moxibustion stimulation focusing on the parameters used for EA and the analgesic effect of moxibustion. To introduce the unique attempts and procedures by Japanese researchers, we could not exclude references to basic research conducted in other countries to provide a comparison of the procedures and results obtained that widely recognized the mechanisms of pain suppression.

\section{Action of Endogenous Opioid Peptides in the Central Nervous System and Peripheral Tissues in AA}

In the initial stage of basic research into AA in Japan, the similarity to the phenomenon of animal hypnosis that was produced by monotonous low-frequency electrical stimulation was indicated. Takeshige and his colleagues $(11,12)$ recorded electrical activities in conscious rabbits and compared the EEG changes during inversion, pressure application, or low-frequency electrical stimulation through needles. Similar electroencephalogram (EEG) changes were induced by each procedure, and the existence of some neurohumoral factors in this phenomenon was shown by crossed circulation experiments. Later, the central mechanism of animal hypnosis was shown to be different from that of AA, but was modified by the common pathway of AA involving an endogenous opioid factor (13). Takeshige et al. conducted numerous behavioral, electrophysiological and pharmacological experiments in rats using focal brain lesions, electrical stimulation and recording methods. The parameter of EA that they used was low-frequency $(1-2 \mathrm{~Hz})$ stimulation with relatively low intensity and evoking muscle twitch, accompanied by current pulse stimulus that was used as an index of intensity.

The central mechanisms of AA that they elucidated are summarized as follows: low-frequency EA to the acupoint activate AA pathways in the spinal cord, which ascend into the contralateral anterolateral tract (ALT) through the dorsal part of the periaqueductal gray (PAG), lateral hypothalamus (LH) and medial hypothalamic arcuate nucleus (M-HARN) (14). Furthermore, the pituitary gland is activated through the preoptic area (POA) and median eminence (ME) (15). Humoral factors ( $\beta$-endorphin and dopamine) are released from the pituitary gland. These humoral factors are essential for connection between the afferent and efferent pathways in AA (from M-HARN to the posterior hypothalamic arcuate nucleus (P-HARN) $(16,17)$. An efferent pathway passes through the hypothalamic ventromedian nucleus (HVM) then divides into two descending pain inhibitory systems in the brain stem (18). One is a serotonergic pain inhibitory system in the raphe dorsalis and raphe magnus. The other is a noradrenergic system in the reticular paragigantocellular nucleus. These pain inhibitory systems descend into the dorsolateral funiculus (DLF) and block the input of pain sensation in the spinal dorsal horn.

Figure 1 schematically demonstrates their concept of neural substrates in the central nervous system and endogenous opioids on AA produced by low-frequency EA to acupoints. Functional roles of the PAG, arcuate nucleus of hypothalamus and descending inhibitory systems in AA have been well recognized (19), although the role of the pituitary gland in AA remains controversial (20). Endogenous opioids responsible for EA analgesia have been intensely investigated by Han's laboratory. Essentially, three types of endogenous opioids and receptors have been shown to participate in a frequency-dependent manner. The relationships between EA analgesia and endogenous opioids are summarized as follows: the low-frequency EA $(2 \mathrm{~Hz}$ or $2-15 \mathrm{~Hz})$ activates $\mu$ and $\delta$ opioid receptors via the release of enkephalin, $\beta$ endorphin and endomorphins in the supraspinal parts of the central nervous system and high-frequency 


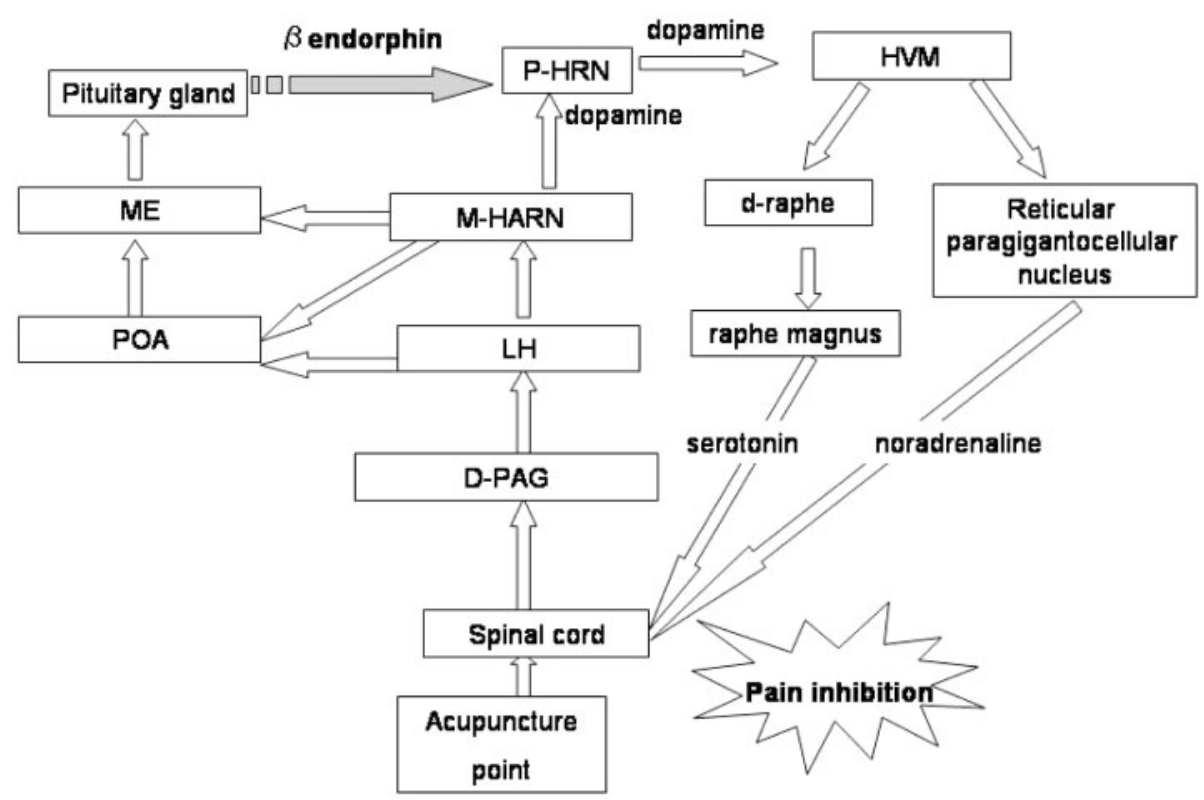

Figure 1. Schematic of the central mechanism of AA. Low-frequency acupuncture stimulation to the acupoint activates AA afferent pathways in the spinal cord, which ascend into dorsal periaqueductal gray (D-PAG), LH and (M-HARN) through the contra-anterolateral tract. Furthermore, the pituitary gland is activated through the POA and ME. Humoral factor(s) ( $\beta$-endorphin) is released from the pituitary gland. This humoral factor is essential for connection between the afferent and efferent P-HARN pathways for AA. The efferent pathway passes through the (HVM) and divides into two descending pain inhibitory systems in the brain stem. One is a serotonergic pain inhibitory system in the raphe dorsalis and raphe magnus. The other is a noradrenergic pain inhibitory system in reticular paragigantocellular nucleus. Two descending pathways in the DLF block the input of pain sensation in the spinal dorsal horn. [Modified from Takeshige et al. (11-18)].

stimulation $(100 \mathrm{~Hz})$ activates $\kappa$ opioid receptors via the release of dynorphin in the spinal cord $(21,22)$. Individual differences between subjects receiving AA are also an important issue. It is also known that the released endogenous opioids (especially enkephalin) are rapidly degraded by enzymes such as carboxypeptidase or leucine aminopeptidase. Kitade demonstrated that administration of D-phenylalanine (a blocker of carboxypeptidase) enhanced the naloxone-reversible electro acupuncture analgenic (EAA) in humans (23). The differences of enzymatic activity between subjects is proposed as a plausible explanation for the existence of EA responders and non-responders. Recently, endogenous peptides such as CCK-8 and nociceptin were found to have a role as anti-AA substances $(24,25)$. On the other hand, the endogenous opioids were released not only in the central nervous system, but also into the peripheral blood. $\beta$-Endorphin, but not ACTH was increased in plasma by low-frequency $(3 \mathrm{~Hz})$ EA stimulation to LI4 (Hoku)ST36 (Zsusanli) accompanied by an increase in the thermal pain threshold of abdominal skin in human (26).

The increased plasma $\beta$-endorphin might have produced an effect in the peripheral nervous system because it could not pass through the blood-brain barrier. Recently, Sekido reported the role of the peripheral opioid receptor in AA using hyperalgesic experimental animals. Anti-nociceptive behavior elicited by $3 \mathrm{~Hz}$ EA was almost completely antagonized by an intraperitoneal injection of naloxone in normal rats, but not in rats with carrageenan-induced inflammation. On the other hand, direct injection of naloxone to inflamed tissues antagonized AA in a dose-dependent manner (27). These peripheral opioid actions might be triggered by release of corticotropin-releasing factor (CRF) or interleukin-1 $\beta$ elicited by low-frequency EA stimulation (28).

These findings indicate that peripheral endogenous opioidergic mechanisms activated by EA stimulation included the immune system in regulating nociception in pathological conditions (Fig. 2). Long-lasting analgesia is one of the features of EA producing endogenous opioids. Hashimoto et al. (29) reported that manual acupuncture (twisting at $1 \mathrm{~Hz}$ ) induced both long-lasting and short-term inhibitory effects on nociceptive discharges in spinal neurons. Long-lasting inhibition involves endogenous opioids through the descending pain inhibitory system, but short-term inhibition remained after a complete spinal lesion at the cervical level or systemic administration of naloxone (30). These findings suggest that non-opioidergic and proprio-spinal pain inhibitory processes might also be involved in AA.

\section{Electrophysiological Investigations of DNIC-like Analgesic Action Mechanisms of Acupuncture and Moxibustion}

In Japan, moxibustion treatment has been used frequently as a form of acupuncture, but little is known in basic study, especially of its analgesic action. 


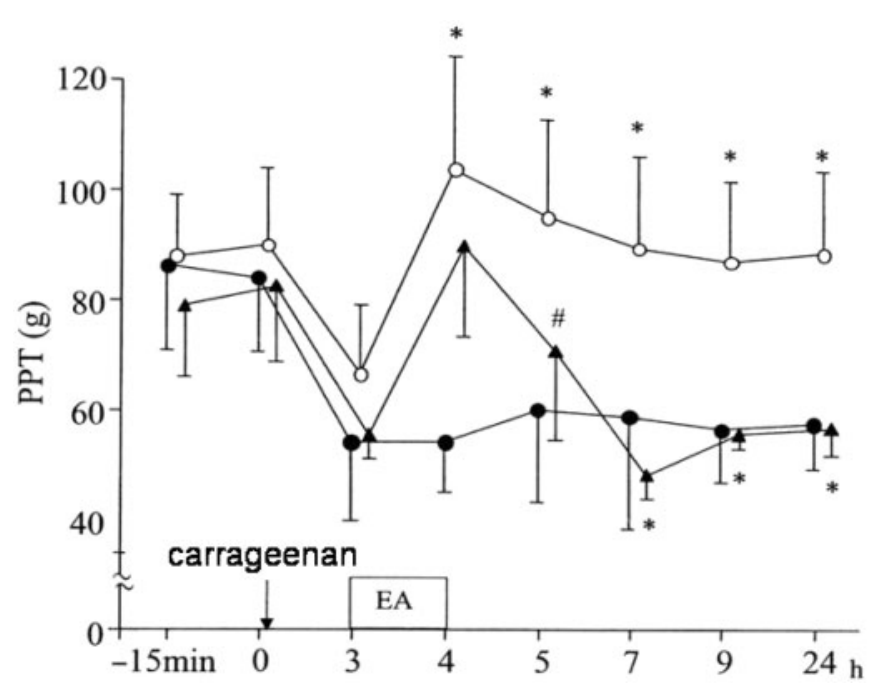

Figure 2. Immunosuppression by cyclosporin A on EAA. Solid circles: control rats (carrageenan injection only, $n=6$ ), open circles: vehicle + EA $(n=6)$. Closed triangles: Cyclosporin A (CsA: immunosuppressant) was injected intraperitoneally 48 and $24 \mathrm{~h}$ before carrageenan administration into the subcutaneous intraplantar of the hindpaw $(n=6)$. CsA clearly inhibited long-lasting analgesia. [Modified from (51) Bull Meiji Univ Oriental Med, 2002].

Moxibustion therapy involves various procedures including the direct burning of small moxa on the skin, indirect burning interposed by various materials (including salt, various plant leafs, ginger slices, garlic slices) and burning above the skin. In this review, moxibustion was considered to be a thermal stimulation to a particular sensory receptor, so direct and indirect moxa (interposed by air) were not differentiated. Murase et al. (31) reported that applying moxibustion to the hind limb of rats clearly produced suppressive effects on trigeminalevoked discharge as well as manual acupuncture stimulation and suggested a close relationship with the DNIC-like mechanism for analgesia (Fig. 3).

DNIC is an analgesic phenomenon where neural activities of convergent neurons in the spinal or medullary dorsal horn are inhibited by noxious stimuli applied to remote areas of the body. This phenomenon was assumed as a possible neural mechanism of counterirritation, i.e. noxious input suppresses the pain transmission. Bing (32) clearly demonstrated that manual acupuncture to the Zusanli (ST36) acupoint-induced, DNIC-like suppression and suppression of pain due to immersion in hot water, and that both suppressions were partially antagonized by naloxone. The central mechanisms of DNIC phenomena have suggested a role for the subnucleus reticularis medulla (SRD) (33) and descending inhibitory mechanisms (34). On the other hand, Sumiya demonstrated the role of nucleus submedius $(\mathrm{Sm})$ in the DNIC-like suppression of viscero-somatic reflex electromyogram (EMG) activities elicited by colorectal distension in anesthetized rats. Manual acupuncture to the cheek or limb clearly suppressed the reflex EMG
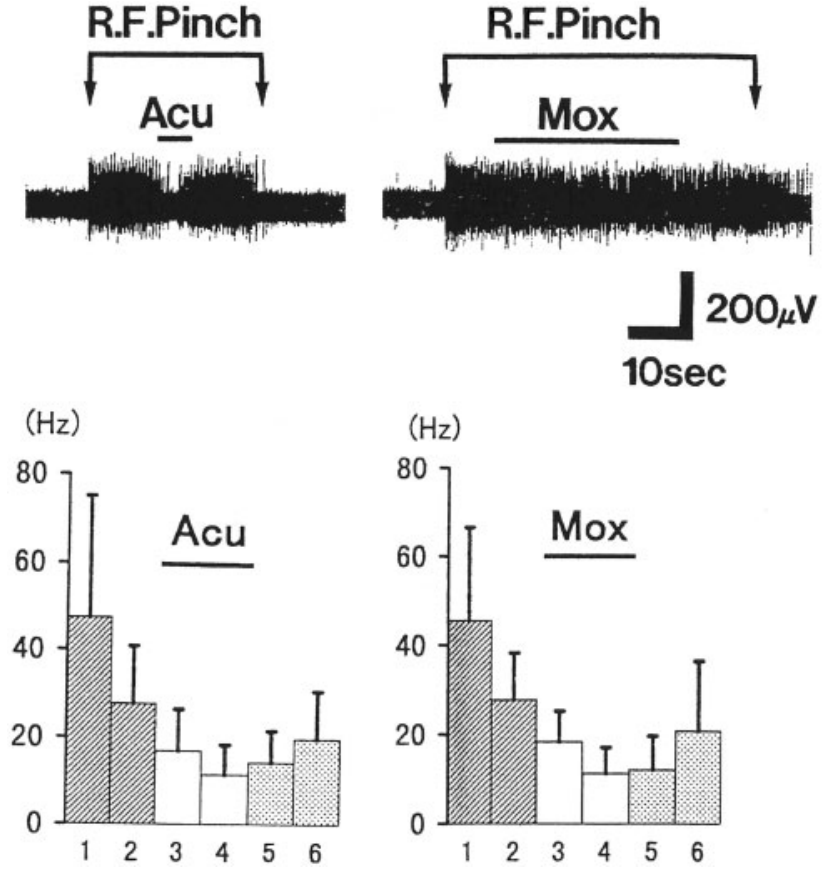

Figure 3. Suppressive effects of acupuncture and moxibustion on the evoked neural discharges of caudalis neurons. (A) Acupuncture (Acu) and moxibustion (Mox) stimuli were applied to the hindpaw during pinch-evoked discharges from trigeminal receptive fields. (B) Averaged histograms of spike frequency of 17 caudalis neurons (mean \pm standard deviation). Histograms 1-2, 3-4 and 5-6 indicate the mean discharges before, during and after conditioning stimuli, respectively. [Modified from (31) Jpn J Physiol, 2000].

activities, and this inhibition was completely abolished by focal injection of lidocaine bilaterally into Sm nuclei, whereas saline injection had no effect (35).

Activation of thin afferent fibers (A- $\delta$ and C- fibers) is required for the development of the DNIC phenomena. DNIC is provoked by not only noxious mechanical stimulation like pinch or manual acupuncture, but also by heat stimulation such as moxibustion, or injection of hypertonic saline into muscle (36). So possible afferent inputs for DNIC are a type of nociceptor responsive to mechanical, thermal and chemical stimuli, and are distributed through the skin, muscle and viscera of the entire body. It is interesting to note that DNIC-like inhibitory effects on nociceptive responses were produced by acupuncture needle manipulation of the muscle rather than cutaneous tissue (36). This manipulation technique produces heavy, dull and deep pain sensations that are often referred. These typical sensations are known as 'de-qi' and are clinically regarded as an important indicator in the production of acupuncture effects.

\section{Afferent Fibers Responsible for Acupuncture and Moxibustion in Analgesic Actions}

It was clearly demonstrated that various somatosensory afferent receptors were activated by manual acupuncture 


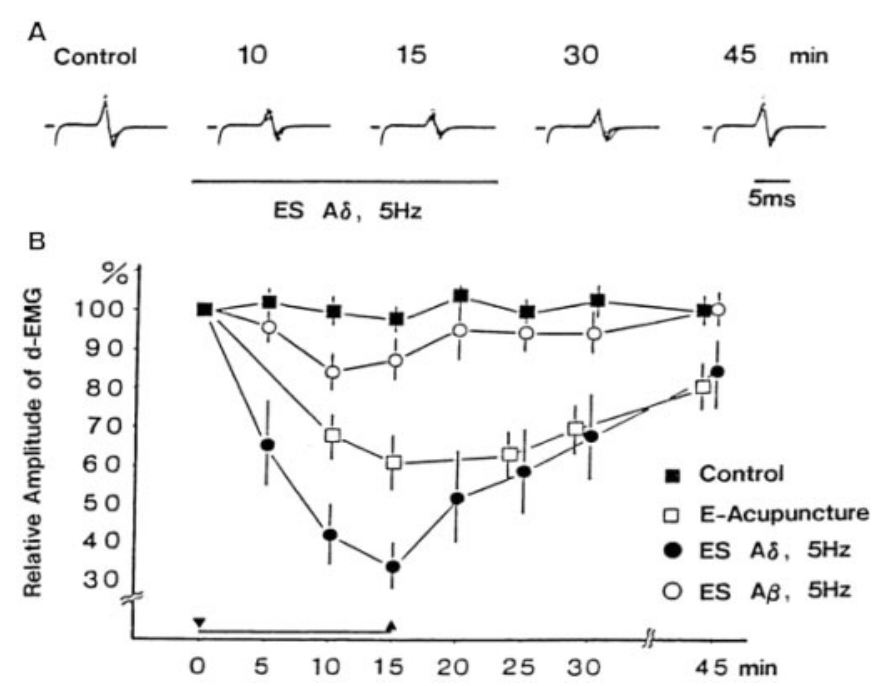

Figure 4. Selective conditioning stimulation of the A- $\delta$ fiber suppressed the JOR. (A) Typical examples of JOR suppression by A- $\delta$ fiber stimulation with triangular stimulus pulses $(5 \mathrm{~Hz}, 15 \mathrm{~min})$. (B) Comparison of suppressive effects induced by conditioning A- $\delta$ fiber, A- $\beta$ fiber and EA stimulation. (44, Exp Neurol, 1982).

using single-fiber recording in animals (37,38). Synchronizing burst discharges to manual twisting of the acupuncture needle at $1 \mathrm{~Hz}$ were shown in fastconducting afferent fibers, whereas lower discharges of the slower fiber group were also elicited by the same stimuli. These results suggest that different fiber groups activated during acupuncture stimulation might produce various effects in central nervous system (CNS). To clarify which afferent fiber is responsible for inducing the $d e-q i$ sensation, microneurography is undoubtedly a useful method. Recordings of neural discharges from human subjects make it possible to investigate the quality of the subjective sensation and their responsible afferent fibers. A close relationship between the particular de-qi sensation and the excitation of thin afferent fibers responsive to manual acupuncture has been demonstrated, and the frequency of discharges was consistent with the intensity of the de-qi sensation (39).

The jaw-opening reflex (JOR) evoked by tooth pulp stimulation is assumed to be a kind of nociceptive reflex in animal experiments. Toda (40) clearly demonstrated that suppression of the JOR elicited by EA $(45 \mathrm{~Hz})$ was related to the amplitude of A- $\beta$ component in the compound action potentials. It was also demonstrated that one of the main sites of EA action on JOR is in the caudal part of the spinal trigeminal nucleus, which is the second-order neuron level in the trigeminal noxious sensory pathway $(41,42)$. This analgesic action of thick A- $\beta$ afferent fibers was considered as involvement of the peripheral pain inhibitory mechanism known as the gate control hypothesis (43).On the other hand, selective activation of the A- $\delta$ fibers in the peripheral nerve by triangularly shaped stimulus pulses and EA at low frequency $(5 \mathrm{~Hz})$ produced a significant suppression of the JOR in rats, whereas that of the maximal excitation of A- $\beta$ fibers by the square-wave pulses had no effect at $5 \mathrm{~Hz}$ (44) (Fig. 4). This finding clearly indicates the inhibitory effects of A- $\delta$ afferent fibers, instead of A- $\beta$ fibers at low frequency, are involved in EAA. Moreover, topical application of capsaicin on the nerve trunk for the elective blockade of the conduction by thin (A- $\delta$ and $\mathrm{C}$ ) afferent fibers was used by Okada and clear abolishment of the JOR suppression produced by manual acupuncture and heat stimuli to hind paw was demonstrated (45). Similar findings demonstrated that capsaicin-sensitive fibers are responsible for the EA analgesia (46). Capsaicin is well known as a potent neurotoxin of the nociceptive primary sensory neurons. Systemic capsaicin treatment during the neonatal period prolonged tail flick latency (increase pain threshold), but could not produce EA analgesia in rats (47). Participation of moxibustionsensitive afferents must be considered responsible for AA. Not only acupuncture, but also moxibustion have been used in clinical situations for over 2000 years based on the same meridian theory.

Moreover, a survey of the ancient medical literature found in the Mawangdui (馬王堆) tomb in Changsha, China, clearly demonstrated that the concept of meridians was established by clinical experience of moxibustion therapy and that no acupuncture treatment existed when the meridian concept was initiated (48).We cannot completely exclude the roles of thick afferent fibers in the production of analgesia via gate control mechanisms; however, mechanoreceptors innervated by thick fibers cannot be activated by thermal stimuli such as moxibustion. It should be stressed that the fundamental mechanism of acupuncture and moxibustion is based on moxibustionsensitive input. Polymodal types of nociceptors that respond to mechanical, thermal and chemical stimulation are particularly sensitive to capsaicin, and innervated by the afferent $\mathrm{C}$ fibers in the skin, as well as by both A- $\delta$ and $\mathrm{C}$ fibers in the muscle. These polymodal receptors seem to be the most plausible candidates for acupuncture and moxibustion; they are not purely nociceptors, but responsive to non-noxious stimuli such as scratching the skin. This functional characteristic of polymodal receptors may be important for further investigations of the comfortable nature of acupuncture and moxibustion that has not been intensively studied until now.

\section{Conclusions}

In this brief review we have introduced the experimental research of acupuncture and moxibustion-mediated analgesia, focused on that conducted by the Japanese researchers. Analgesic effects of acupuncture have been well clarified by experimental studies and the participation of various endogenous opioids and their receptors has been widely accepted (Fig. 5). However, this seems to 


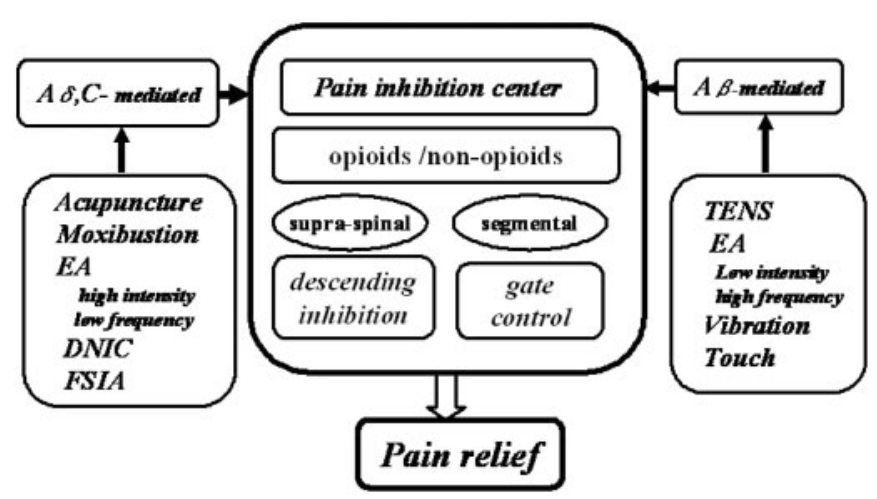

Figure 5. Schematic diagram of the endogenous pain inhibitory system produced by acupuncture, moxibustion and other procedures including EA.

be inadequate for explaining the acupuncture action on patients suffering pain, because the stimulus conditions in animal experiments are much stronger than those used in clinical situations. Several Japanese researchers employed moderate EA and they also induced analgesic effects. Discrepancies between the results may be explained by differences in the EA parameters. The general procedures of Japanese-style acupuncture and moxibustion are gentle and sometimes induce comfortable sensations, although they are nociceptive in nature. Moreover, careful palpation for detecting the tenderness and palpable hardenings under the skin are considered extremely important by experienced acupuncturists (49). The importance of the moxibustion should be noticed, since recent archaeological investigation has demonstrated the essential role of moxibustion therapy in the development of the Chinese meridian theory. Moreover, the nature and development of acupoints remains as an important issue to be resolved by acupuncture and moxibustion research. Sensitization of the polymodal receptors has been proposed as a rational explanation of the nature of acupoint formation. However, more concrete evidence is required from future investigations (50).

\section{References}

1. Ma SX. Neurobiology of acupuncture: toward CAM. Evid Based Complement Alternat Med 2004;1:41-7.

2. Usichenko TI, Ma SX. Basic science meets clinical research: 10th North American symposium on acupuncture: Sheraton Fisherman's Wharf Hotel, San Francisco, USA, July 1, 2004. Evid Based Complement Alternat Med 2004;1:343-4.

3. Fan Q, Zhou J. Electro-acupuncture in relieving labor pain. Evid Based Complement Alternat Med 2007;4:125-30.

4. Liu HX, Tian JB, Luo F, Jiang YH, Deng ZG, Xiong L, et al. Repeated $100 \mathrm{~Hz}$ TENS for the treatment of chronic inflammatory hyperalgesia and suppression of spinal release of substance $\mathrm{P}$ in monoarthritic rats. Evid Based Complement Alternat Med 2007;4:65-75.

5. Han JS. Acupuncture and endorphins. Neurosci Lett 2004;361:258-61.

6. Research Group of Acupuncture Anesthesia, Peking Medical College. Effect of acupuncture on pain threshold of human skin. Natl Med J China 1973;3:151-7.
7. Research Group of Acupuncture Anesthesia, Peking Medical College. The role of some neurotransmitters of brain in AA. Sci Sin 1974;17:112-30.

8. Aikawa S. Acupuncture anesthesia and physiology of pain. Keio $J$ Med 1973;50:463-78(in Japanese).

9. Chang HT. Integrative action of thalamus in the process of acupuncture for analgesia. Sci Sin 1973;16:25-60.

10. Yano T, Maruyama A, Tanaka A, Katayama K, Mori K. The effects of electro-acupuncture and TENS on EEG topogram. Bull Meiji Univ Oriental Med 1985;1:55-64(in Japanese).

11. Luo CP, Takeshige C. Similarity of EEG changes induced by inversion, pressure on body parts and peripheral low frequency stimulation. J Showa Med Assoc 1977;37:279-86(in Japanese).

12. Luo CP, Takeshige C. Neurohumoral influences of inversion, pressure on body parts and peripheral low frequency stimulation of electrical subcortical activities with crossed circulation. J Showa Med Assoc 1977;37:273-7(in Japanese).

13. Takeshige C. Analgesia producing mechanism in acupuncture anesthesia. Nippon Seirigaku Zasshi 1987;49:83-105(in Japanese).

14. Sato T, Takeshige C, Shimizu S. Morphine analgesia mediated by activation of the AA-producing system. Acupunct Electrother Res 1991;16:13-26.

15. Takeshige C, Zhao WH, Guo SY. Convergence from the preoptic area and arcuate nucleus to the median eminence in acupuncture and nonacupuncture point stimulation analgesia. Brain Res Bull 1991;26:771-8

16. Takeshige C, Tsuchiya M, Guo SY, Sato T. Dopaminergic transmission in the hypothalamic arcuate nucleus to produce AA in correlation with the pituitary gland. Brain Res Bull 1991;26:113-22.

17. Takeshige C, Oka K, Mizuno T, Hisamitsu T, Luo CP, Kobori M, et al. The acupuncture point and its connecting central pathway for producing AA. Brain Res Bull 1993;30:53-67.

18. Takeshige C, Sato T, Mera T, Hisamitsu T, Fang J. Descending pain inhibitory system involved in AA. Brain Res Bull 1992;29:617-34.

19. Stux G, Pomeranz B. Basics of Acupuncture. Berlin: SpringerVerlag, 2003

20. Fu TC, Halenda SP, Dewey WL. The effect of hypophysectomy on acupuncture analgesia in the mouse. Brain Res 1980;202:33-9.

21. Han JS, Terenius L. Neurochemical basis of AA. Annu Rev Pharmacol Toxicol 1982;22:193-220.

22. Han JS. Acupuncture: neuropeptide release produced by electrical stimulation of different frequencies. Trends Neurosci 2003;26:17-22.

23. Kitade T, Odahara Y, Shinohara S, Ikeuchi T, Sakai T, Morikawa $\mathrm{K}$, et al. Studies on the enhanced effect of AA and acupuncture anesthesia by D-phenylalanine (first report) - effect on pain threshold and inhibition by naloxone. Acupunct Electrother Res 1988;13:87-97.

24. Shou Y, Sun YH, Shen JM, Han JS. Increased release of immunoreactive CCK-8 by electroacupuncture and enhancement of electroacupuncture analgesia by CCK-B antagonist in rat spinal cord. Neuropeptides 1993;24:13-144.

25. Zhu CB, Xu SF, Cao XD, Wu GC, Zhang XL, Li MY, et al. Antagonistic action of orphanin FQ on acupuncture analgesia in rat brain. Acupunct Electrother Res 1996;21:199-205.

26. Ishimaru K. Effects of AA on post-operative pain after abdominal surgery and the relationship of endogenous analgesic substances. Bull Meiji Univ Oriental Med 2000;26:11-22(in Japanese).

27. Sekido R, Ishimaru K, Sakita M. Differences of electroacupuncture-induced analgesic effect in normal and inflammatory conditions in rats. Am J Chin Med 2003;31:955-65.

28. Sekido R, Ishimaru K, Sakita M. Corticotropin-releasing factor and interleukin-1 $\beta$ are involved in the electroacupuncture-induced analgesic effect on inflammatory pain elicited by carrageenan. Am J Chin Med 2004;32:269-79.

29. Hashimoto T. Analgesia induced by manual acupuncture: its potency and implication. Kitasato Arch Exp Med 1993;65:73-82.

30. Hashimoto T, Aikawa S. Manual acupuncture and its central mechanisms: involvement of propriospinal and descending pain inhibitory system. JSAM 1994;44:181-90.

31. Murase K, Kawakita K. Diffuse noxious inhibitory controls in antinociception produced by acupuncture and moxibustion on trigeminal caudalis neurons in rats. Jpn J Physiol 2000;50:133-40. 
32. Bing Z, Villanueva L, Le Bars D. Acupuncture-evoked responses of subnucleus reticularis dorsalis neurons in the rat medulla. Neuroscience 1991;44:693-703.

33. Bouhassira D, Villanueva L, Bing Z, le Bars D. Involvement of the subnucleus reticularis dorsalis in diffuse noxious inhibitory controls in the rat. Brain Res 1992;595:353-7.

34. Villanueva L, Le Bars D. The activation of bulbo-spinal controls by peripheral nociceptive inputs: diffuse noxious inhibitory controls. Biol Res 1995;28:113-25.

35. Sumiya E, Kawakita K. Inhibitory effects of acupuncture manipulation and focal electrical stimulation of the nucleus submedius on a viscerosomatic reflex in anesthetized rats. Jpn $J$ Physiol 1997;47:121-30.

36. Hashimoto T, Aikawa S. Manual acupuncture and its peripheral mechanisms: involvement of nociceptors in muscle. JSAM 1994;44:191-200.

37. Ikeda H, Uchida S, Simura M, Suzuki A, Aikawa Y. Single afferent nerve fibers in the spinal dorsal roots activated by manual acupuncture needle stimulation in frog's hind limbs. JSAM 2001;51:91-7.

38. Kagitani F, Uchida S, Hotta H, Aikawa Y. Manual acupuncture needle stimulation of the rat hindlimb activates groups I, II, III and IV single afferent nerve fibers in the dorsal spinal roots. Jpn $J$ Physiol 2005;55:149-55.

39. Kawakita K, Gotoh K. Role of polymodal receptors in the acupuncture-mediated endogenous pain inhibitory systems. In: Kumazawa T, Kruger L, Mizumura K (eds). The Polymodal Receptor-A Gateway to Pathological Pain. Amsterdam: Elsevier; 1996, 507-23.

40. Toda K. Effects of electro-acupuncture on rat jaw opening reflex elicited by tooth pulp stimulation. Jpn J Physiol 1978;28:458-97.
41. Toda K, Iriki A, Tanaka H. Jaw opening reflex affected by electroacupuncture in rat. Neurosci Lett 1981;25:161-6.

42. Toda K. Changes of the jaw opening reflex activity by electroacupuncture stimulation in rat. $\mathrm{Am} J$ Chin Med 1981;9:236-42.

43. Melzack R, Wall PD. Pain mechanisms: a new theory. Science 1965;150:971-9.

44. Kawakita K, Funakoshi M. Suppression of the jaw-opening reflex by conditioning A-delta fiber stimulation and electro-acupuncture in the rat. Exp Neurol 1982;78:461-5.

45. Okada K, Oshima M, Kawakita K. Examination of the afferent fiber responsible for the suppression of jaw-opening reflex in heat, cold, and manual acupuncture stimulation in rats. Brain Res 1996;740:201-7.

46. Kawamura H, Ninomiya Y, Funakoshi M. Effects of local application of capsaicin to peripheral nerves on electro-AA for each part in the rat. JSAM 1996;46:65-9(in Japanese).

47. Kawamura H, Ninomiya Y, Funakoshi M. Electro-AA after neonatal and adult capsaicin treatment. JSAM 1996;46:1-6(in Japanese).

48. Harper D. Early Chinese Medical Literature: The Mawangdui Medical Manuscripts. London: Kegan Paul International, 1998.

49. Kawakita K, Okada K, Kawamura H. Analysis of a questionnaire on the characteristics of palpable hardenings: a survey of experienced Japanese acupuncturists. JAM(Online Journal of JSAM) 2005;1:1-8.

50. Kawakita K, Okada K. Mechanisms of action of acupuncture for chronic pain relief - polymodal receptors are the key candidates. Acupunct Med 2006;24 (Suppl): S58-66.

Received July 7, 2006; accepted July 6, 2007 


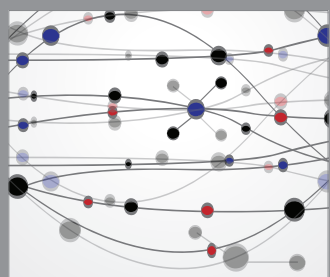

The Scientific World Journal
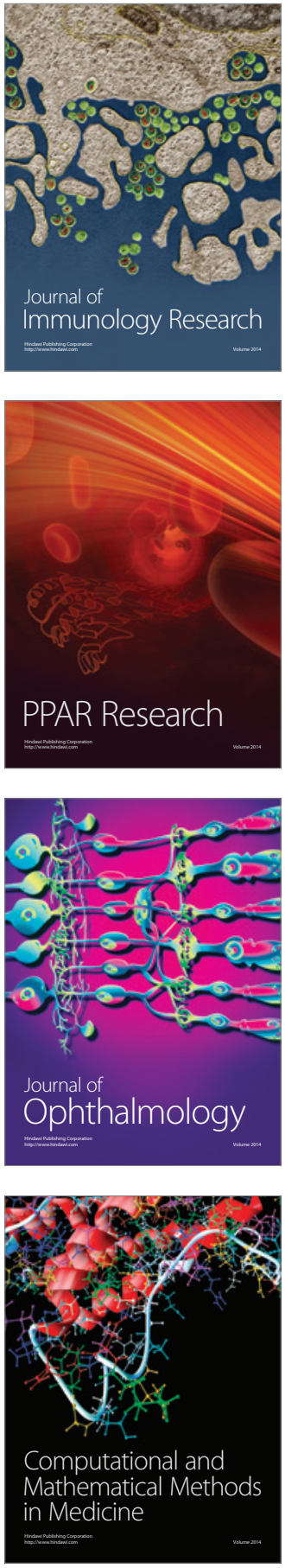

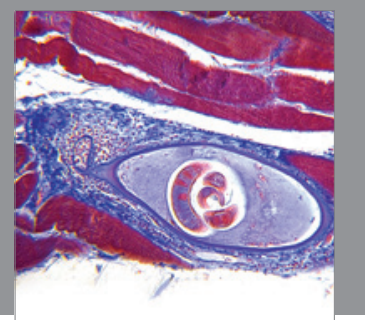

Gastroenterology

Research and Practice
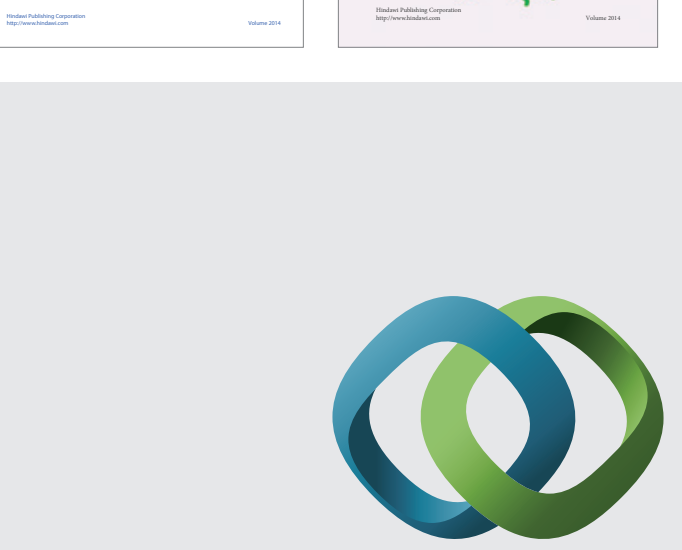

\section{Hindawi}

Submit your manuscripts at

http://www.hindawi.com
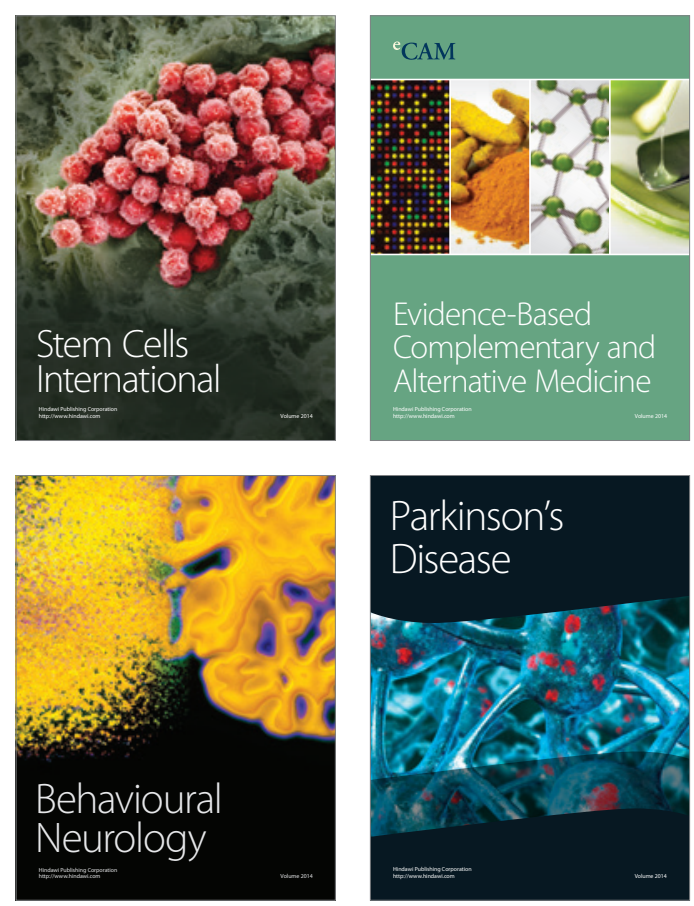

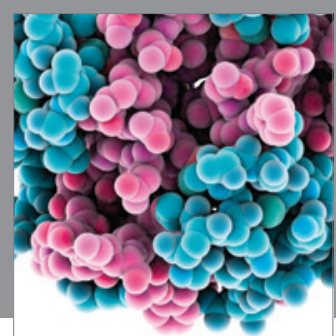

Journal of
Diabetes Research

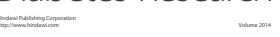

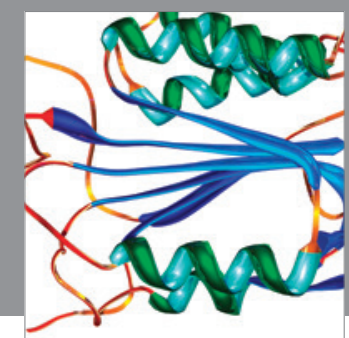

Disease Markers
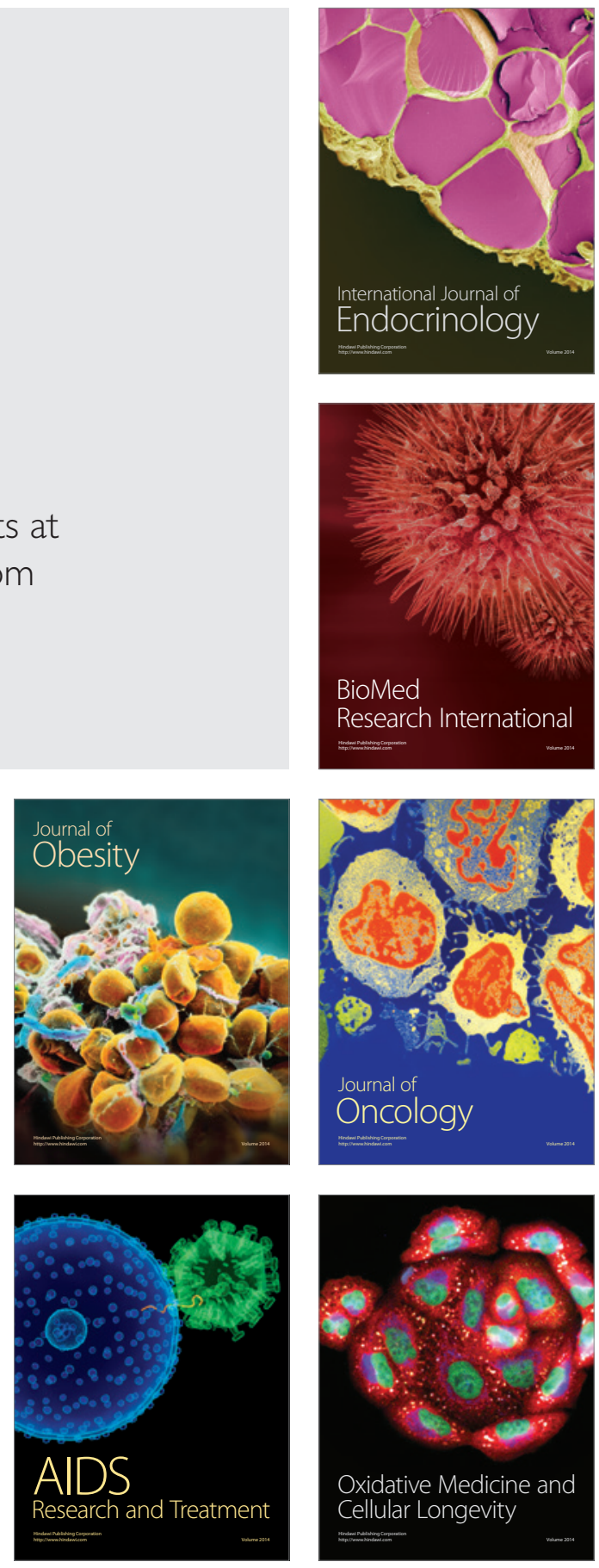